\title{
Computer Simulation of Stenotic Carotid Bifurcations Hemodynamic and Ultrasonography
}

\author{
Wei $\mathrm{Li}^{*}{ }^{1}$ and Qinghua Yang ${ }^{2}$ \\ ${ }^{1}$ Computer School, China West Normal University, Nanchong, Sichuan, 637002 China \\ ${ }^{2}$ Medical Imaging Department, North Sichuan Medical College, Nanchong, Sichuan, 637000 China
}

\begin{abstract}
The carotid sinus of the carotid artery (CA) bifurcation is one of the favored sites for the genesis and development of atherosclerotic lesions. The direct reason is carotid arteries bifurcation and stenoses may lead to great flow pattern change. Aim of this article is to investigate the effect of different eccentric stenosis of internal carotid artery on blood flow. The blood flow in artery is simulated numerically and the simulation is based on convicted reasonable of vascular profile and flow environment in vivo. The simulation or the computer flow dynamic calculation reveals the eccentric stenosis can make great effort to the flow pattern. And more serious stenosis proliferation can be generated. Doppler ultrasound (DUS) are widely used in blood flow detection for the diagnosis of cardiovascular diseases (CAD). It is the gold standard of the diagnosis of vessel related diseases and the color sonography display is familiar for clinicians and internist, we simulated the color sonography of stenosis carotid arteries bifurcation mocked the DUS display method. The results are proved to be visual, detailed and accurate that it can more directly use in clinic. All simulation above can alleviate the effects of measurement errors and can be used as the complement of ordinary ultrasound measures.
\end{abstract}

Keywords: Carotid Bifurcation, Computer Simulation, Sonography, Hemodynamic.

\section{INTRODUCTION}

Occupying diseases of vascular, such as atherosclerosis, stenosis, abdominal aortic aneurysm are widely and seriously diseases today. For in vitro monitoring of vascular anatomy, several medical imaging modalities are used such as MRI, CT and Ultrasound. But by MRI or CT the blood flow cannot measured accuracy, and always need imaging agency and tedious procedure.

Ultrasound or technology called duplex can provides precision velocity measure inexpensive, safely and conventionally. Color flow Doppler imaging (CFDI) can be used for recruitment qualification, pre-treatment classification and post treatment surveillance for remodeling and restenosis.

Due to the ultrasound technology, such as the complication of blood flow, limitation of sample zone and resolution of transducer, signal processing imprecise, we cannot descript the precise flood flow of each voxel in specially appointed time. Recently, scientist and research prefer integrate computer simulation of Doppler ultrasound and computed flow dynamics (CFD) insight into the complex interaction between the acoustic, signal processing and blood flow dynamics $[1,2]$.

\section{METHODS}

The medical imaging technology measuring the profile of vascular are seemed to be matured today. That means we can

*Address correspondence to these authors at the Computer School, China West Normal University, Nanchong, Sichuan, 637002 China;

Tel: 86-10-56186836; Fax: 86-10-56186836; E-mail: nos036@163.com get more reliable anatomy by different imaging modalities. Patent US8, 315, 814, titled" Method and system for patientspecific modeling of blood flow" provide a method to monitor the function of heart and vascular, it consists of the following steps: 1) the obtaining and preprocessing patientspecific anatomical data; 2) creating the three-dimensional model based on obtained anatomical data; 3 ) preparing the model for analysis and determining boundary conditions; 4) performing the computational analysis and outputting results. It can be used to providing patient-specific treatment planning. On the other side, it can be used to assessing myocardial perfusion and plaque vulnerability [3].

Our procedure is start from the profile of vascular, computing and generating the blood dynamic based on CFD. Comply with ultrasound Doppler imaging method, we change the flow distributes to the color blood exhibition.

\subsection{Geometric Model Construction}

The aim of medical Ultrasound detection is stenosis, thrombosis and occluding and more, the disturbing of blood including the vascular bifurcation and special twisting. We chose the carotid sinus as our simulation modeling for its typical influence of blood field. It was be study more often by past experiment or instrument measure. Both clinical and post mortem studies indicate that, in humans, the carotid sinus of the carotid artery bifurcation is one of the favored sites for the genesis and development of atherosclerotic lesions $[4,5]$. 
Carotid artery supplies blood to brain and neck. It is a Yshaped artery, which can be divided into three parts. First is common carotid artery (CCA) which is bifurcated into two arteries; one internal carotid artery (ICA) which has a sinus and the other one is of smaller diameter called external carotid artery (ECA).

The carotid artery profile are using the classic model establish by Bharadvaj. As the depict of Fig. (1) Xc means CCA and it is the inlet of blood flow. Xi refers to ICA and Xe refers to ECA, they are outlets. The angle between ICA and ECA is 55 degree. SS1, SS2 and up to SS6 are the diameter of each section of ICA. EC1 to EC4 are the diameter of ECA, and $\mathrm{CC} 1, \mathrm{CC} 2$ means that of ICA. We establish the geometry by AutoCAD ${ }^{\circledR}[6]$.

In some recent research, carotid artery $3 \mathrm{D}$ profile can be established by advanced imaging technology such an MRI and X-CT. We segment the profile of vessel in every section, use the profiles pile up and generate a 3D entity through computer graphical method. Due to more convict profile the following up calculate may be more precise. In this paper our research the realize of emphasis on the display and its methodology. A simple model is used instead of.

\subsection{Carotid Artery Stenoses}

Carotid artery stenoses, always can be found in ICA. There are many reasons that cause the narrow of ICA. Overall the stenoses can be divided into two categories: eccentric and concentric. The former are more common and we discuss there.

In Fig. (1) the distance $\mathrm{S} 1$ is fixed and the grade of eccentric stenoses depending on the S2. The stenosis is usually caused by plaques, so we can also express grade of vessel narrowing by the volume of plaques. It can simply define by compare S1 in vascular and diameter. When S1 equal to $\mathrm{S} 2$ that means $50 \%$ carotid artery stenosis. There we consider the different stenosis of $30 \%, 45 \%, 60 \%$ and $75 \%$. They refer to from moderate to severe carotid stenosis $[7,8]$.

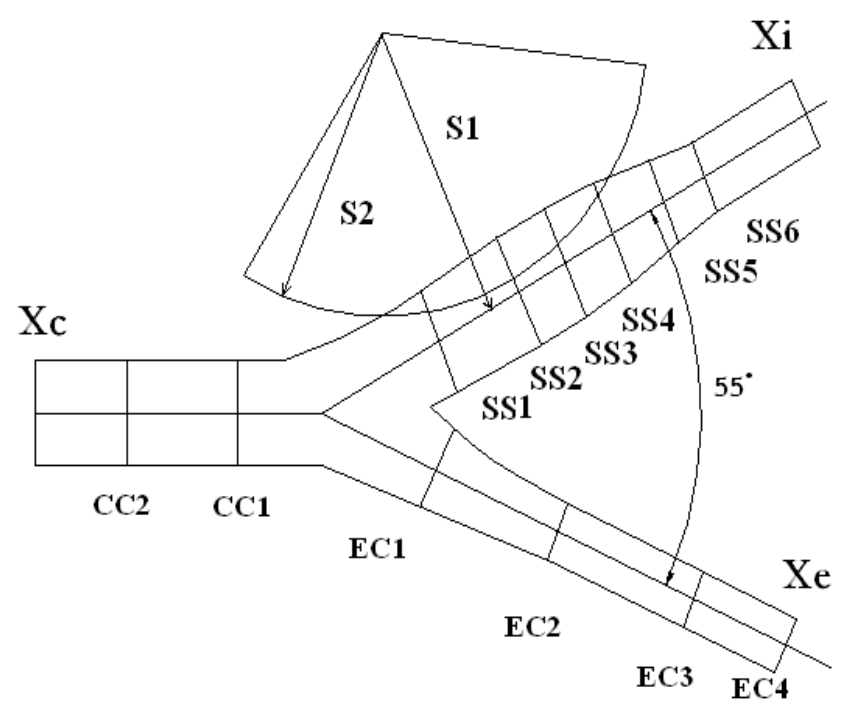

Fig. (1). The carotid artery bifurcation model.
Patent US 7,942,820, titled" Method and system for evaluation of the hemodynamic model in depression for diagnosis and treatment" provides a method for determining the cerebral hemodynamic model for depression using carotid duplex ultrasound to establish percent stenosis of the extracranial right and left internal carotid arteries (RICA, LICA), respectively; and some other site of carotid arteries. It would be of use in a wide range of disease conditions for diagnosis, evaluation of treatment options, effectiveness of surgical measures, and monitoring of progress under certain medications [9].

\subsection{Flow Zone grid and Boundary Define}

Due to the dedicate profile, it is difficult to calculate by manual programming. There the commercially available computer program FLUENT ${ }^{\circledR} 6.1$ was used in the flow simulations. FLUENT ${ }^{\circledR}$ is a finite volume code capable of efficiently solving the governing equations of fluid mechanics. Before the calculation in software, we must grid the flow zone and define the boundary.

Grid are built by the pre-processing software GAMBIT ${ }^{\circledR}$ 2.1 with much denser grids in the adjacent area to the vessel wall where high variable gradients are expected and detailed flow parameters are important. In our simulation the entity is dividing to 43734 and triangular elements were used to mesh faces, Mesh size was nearly equal in all cases.

We set velocity inlet and pressure outlet. The blood vessel considers being rigid wall and the space in wall, inlet outlets is the computer hydraulic zone. All this setting can be convictable in usually condition.

\subsection{Equations Solving}

Governing equations of simulation of blood flow are the Navier-Stokes equations for incompressible viscous fluid flow and the pressure equation:

$\frac{\partial \rho}{\partial t}+\frac{\partial\left(\rho u_{j}\right)}{\partial x_{j}}=0$

$\frac{\partial\left(u_{i}\right)}{\partial t}+\frac{\partial\left(u_{i} u_{j}\right)}{\partial x_{j}}=F_{i}-\frac{1}{\rho} \frac{\partial p}{\partial x_{i}}+\frac{1}{\rho} \frac{\partial \tau_{i j}}{\partial x_{j}}$

where $\rho$ and $\mu$ are, respectively, the density and viscosity of blood, $\mathrm{t}$ is time, $\mathrm{u}=$ ( $\mathrm{ui}, \mathrm{uj}$ ) is the velocity vector, and $\mathrm{p}$ is the pressure. Fi denotes the artificial body force or the feedback signal which is applied at feedback points defined in a feedback domain. The pressure Eq. (2) is derived by substituting the equation of continuity into the divergence of the Navier-Stokes equation, Eq. (1). [10]

\subsection{Blood Flow Field Calculation}

To imitate the same environment that as in vivo, some parameters are list as followings.

Because of ICA is large arteries, so there blood is treated as an incompressible, Newtonian fluid, viscosity is $4.24 \times 10$ $3 \mathrm{~Pa} / \mathrm{ms}$, and a density of $1.0511 \times 103 \mathrm{~kg} / \mathrm{m} 3$. The gauge pressure is $13333 \mathrm{~Pa}(100 \mathrm{~mm} \mathrm{Hg})$ and the flow was assumed 
laminar. The more information about the flow, the calculation or the simulation is more reliable. Accuracy, convenient, dynamic are considered to be preferred and important. The Patent US6,814,702, titled "Apparatus for measuring hemodynamic parameters" given a directed toward an ultrasonography apparatus for measuring and/or monitoring hemodynamic activity, such as blood flow. The present invention comprises a doppler ultrasound unit, one or more transducers, and a portable body. maybe it used for special organs (penile), the similar method can be used to other vascular [11].

Due to the heart pump blood with specific rhythm or pulsatile, the velocity of inlet is fluctuation. That means if we must simulation with sequence cycle to get realistic blood field change. Considering the fluctuation of blood in vivo, and full precise flow field calculate and deficit can be found in The Patent US8,224,640, titled "Method and system for computational modeling of the aorta and heart", it provide a method for multi-component heart modeling and cardiac disease decision support, comprising: generating a multi-component patient specific 4D geometric model of the heart and aorta estimated from a sequence of volumetric cardiac imaging data of a patient generated using at least one medical imaging modality; generating a patient specific 4D computational model based on one or more of personalized geometry, material properties, fluid boundary conditions, and flow velocity measurements in the 4D geometric model; and estimating patient specific material properties of an aortic wall using the 4D geometrical model and the 4D computational model by determining parameters of a constitutive material model of the aortic wall to minimize a residue between a simulated deformation of the aortic wall determined based on a Fluid Structure Interaction (FSI) simulation and a measured deformation of the aortic wall in the 4D geometric model [12].

There we can depict the blood field in given time spot and with specific velocity. In the measure of blood with ultrasound instrumentation, in clinic doctor is inclined to use freeze technology instead of dynamic imaging [13-15]. In our article the inlet velocity is set to $1 \mathrm{~m} / \mathrm{s}$ that are a reasonable peak value that measure by other imaging technology such as ultrasound and MRI.

After the input of some parameters and definition of some situation, the software begins the resolve of NavierStokes equations with finite volume method. Standard pressure discretization and second-order upwind momentum discretization were used. The pressure-velocity coupling scheme used was the semi-implicit method for pressurelinked equation (SIMPLE) algorithm. For uncomplicated model and not exceed 10000 grid the calculation time is no more than 10 minutes. Grid computer, parallel mainframe is widely used in the sophisticated CFD in recent years. That made 4-D real time calculation possible.

\subsection{Ultrasound Display Simulation}

Color Doppler flow imaging (CDFI) is a most used way to analysis the blood flow field. The pseudo color often used in pulse Doppler technology. It measure the velocity of little zone that can be called sample zone. For different velocity direction we use red or blue color. The quantity is converting to the color hue or brightness. And at the same time, we measure the disturbing degree of blood, use green to express. So in ultimate display the two kinds of color are mixed and the color imaging is formed [16].

In the Patent US8,469,887, titled "Method and apparatus for flow parameter imaging" there present invention is directed to a method and an apparatus for performing pulsedwave spectral Doppler imaging at every color flow range gate location in a two-dimensional (or three-dimensional) region of interest. Spectral processing is necessary to determine the flow parameters. Performing this processing at every color flow range gate location creates the twodimensional image. The method disclosed herein generates two-dimensional images of flow parameters such as peak velocity, pulsatility index, resistance index, etc. With the two-dimensional image, the user immediately observes where the most critical value of the flow parameter occurs and what that value is [17].

To simulation the velocity field of ultrasound, we must decomposition the velocity to two parts, parallel and vertical to the sound beam as the Fig. (2).

Doppler effect can only get by the parallel part. Using the Doppler frequency of transducer (ft, MHx), the speed of sound through water (c, $\mathrm{m} / \mathrm{s}$ ) and the cosine angle of transducer to the direction of flow $\left(\theta,{ }^{\circ}\right)$, the resulting Doppler shifted frequencies (fd, $\mathrm{MHz}$ ) were converted to velocities (v, m/s), via the Doppler equation:

$$
v=\frac{f_{d} \cdot c}{2 \cdot f_{t} \cdot \cos \theta}
$$

In our simulation the ICA is about $30^{\circ}$ to horizontal line and equal $\theta$,it can be used in clinic, so vertical velocity can be discard in Doppler imaging.

As an important medical instrumentation, ultrasound can be considered as golden standard for the diagnosis of soft tissue and vascular. And plenty of new products and patents are emerged these years. The Patent US7,128,713, titled "Doppler ultrasound method and apparatus for monitoring blood flow and hemodynamic"

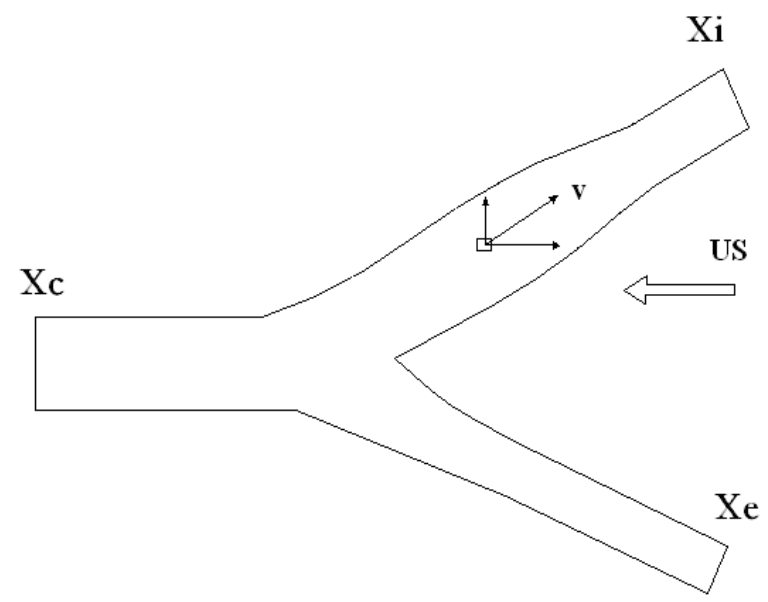

Fig. (2). Velocity decomposition: parallel and vertical component. 
Introduced a new Doppler ultrasound system with advantage in solve these two problems: 1) a complicating factor in locating the ultrasound window is determination of the proper depth at which the desired blood flow is located. 2)Once blood flow has been located, it is usually scanned along the course of the vasculature to determine if there are any localized regions in which there are flow abnormalities, which may indicate various diseases [18].

The velocity distribution is calculated by Fluent as above. But in some case the contour velocity display inconvenient for physicians. That is 1) there no turbulence information in velocity map, we often need two map to study the flow; 2) the color bar are different that means we must reading the color with corresponding numerical mean. We use those data to change display mode so that we can get the ultrasound simulation. The method can use the software Tecplot360 to realize.

After all, the key point of operation including:

1) The color on the screen has three attributes: luminosity, hue and saturation. Luminosity is the degree of brightness or shade of the displayed color; hue is the wavelength (i.e., the actual color displayed, from violet through red), and saturation. These three attributes can be used to produce a variety of color scales. It means we can mix or overlay some parameter display in a picture.

2) The horizontal velocity to display in color, if the value is positive it appears in red. If the value is negative it appears in blue. That stands for situation of the flow (blood red cell) toward or away from the transducers. Flow reversal or low velocity zone may cause to opposite color in display.
3) For some US instrumentation, The variance can be displayed along with the mean frequency by using a red and blue scale with increasing amounts of yellow or green introduced as the variance increases, the more brightness of green means more disturbing happens.

4) Stream lines depict the turbulence sometimes can be added. In condition the flow pattern be great disturbed, it is directly perceived through the transducers

\section{RESULTS AND DISCUSSION}

The procedure as above, after the simulation, formulation we can get the blood flow field in detail. Ultrasound and other imaging technology can only get low resolution imaging with high noise and even with artifacts. This present situation may caused by defect of imaging technology itself. But to get much more reality parameters we can use them $[19,20]$.

The result of simulation can divide into two sections.

\subsection{Blood Velocity Calculates and Display}

Blood flow field calculation is time consumption work with more grids divided. Fig. (3) stand for the velocity distribute of different eccentric stenosis. The color bars of display actual an other kind pseudo-color display mode.

From the pictures of Fig. (3) we can see:

At every vessel the velocity in center is much greater than that near wall. The ICA stenosis can cause the blood velocity boosting. And the trend is more obviously when the stenosis is seriously developed. In classic hemodynamic
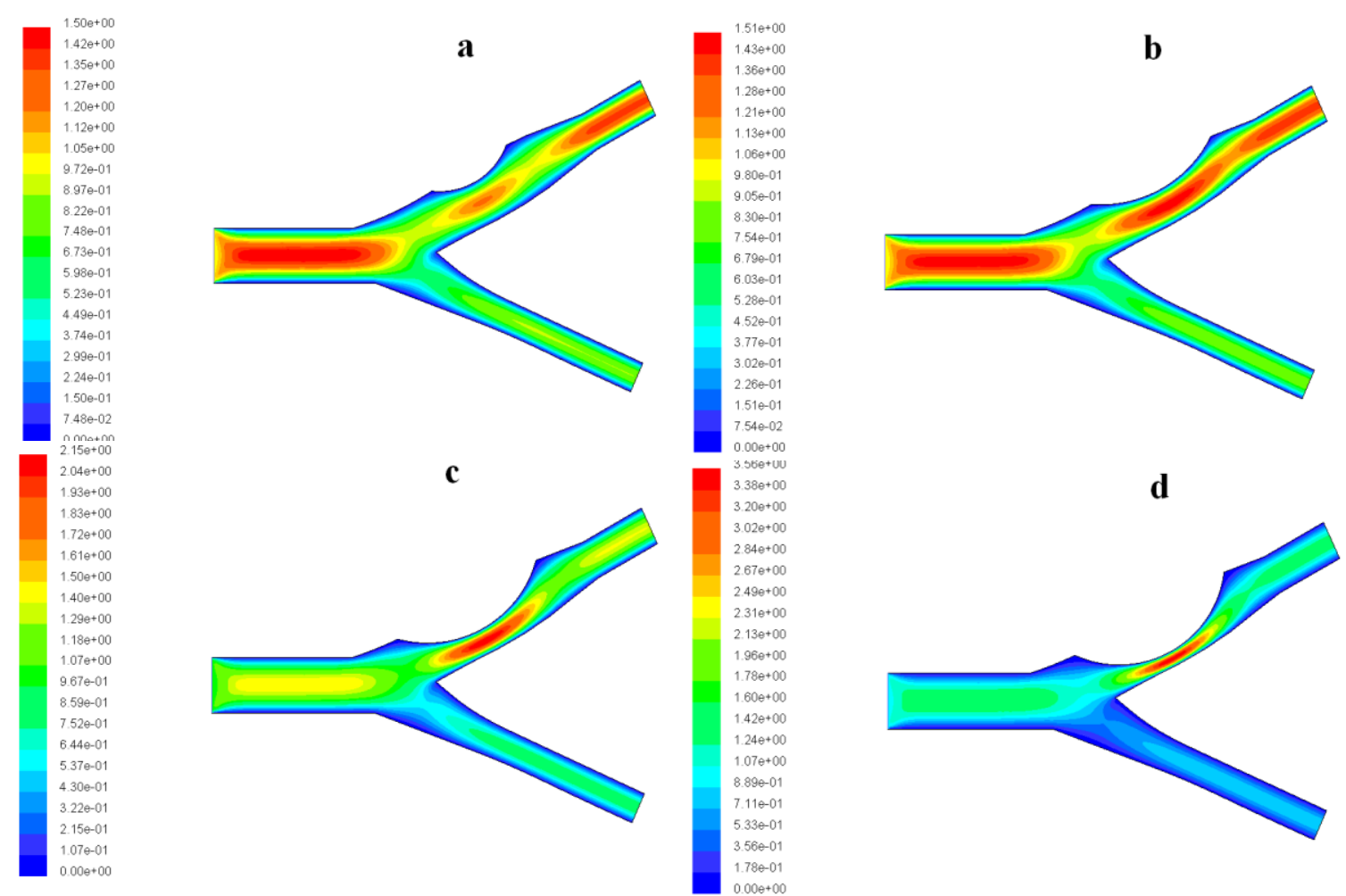

Fig. (3). Contour of velocity in bifurcation a $30 \%$ stenosis. b $45 \%$ stenosis. c $60 \%$ stenosis. d $75 \%$ stenosis. 
analysis or other dynamics viewpoint that can cause an addition force or pressure in vessel wall. That cause deterioration or cause the stenosis more serious.

The velocity boost may change the distribution of blood to two outlets: ICA and ECA. In 30\% stenosis bifurcation the velocity of ECA can maintain, but with the stenosed serious especially that of $75 \%$, the blood in ECA dropped greatly. That means stenosis is not only influence the ICA but also can deteriorate the flow in ECA.

Once stenosis generate, it will create some blood stagnate zone adjacent to the stenosis. This static effect is make the profiling of stenosis or make it worsen. As the simulation result reveal, the more serious stenosed, the more big zone of stagnate exist. That is the pathophysiology of vessel stenosis.

\subsection{Flow Display in Color Ultrasound Mode}

Calculate and display above can depict the flow pattern detailed. After the processing or flow field calculation in CFD. There result is data with space information and the vector information. To research scientist we can chose to display the flow velocity, kinetic energy or turbulence information. The display is more flexible and quantification by the selecting of special cross or section or in many special mode. But in clinical, the doctors are more cling to color Doppler ultrasonography or called CDFI. That is we must express the flow information more vivid, visual and simply. In other word we lost some quantity information or it can be say these are ignoring in practice clinic use [21].

The overall data is post-processing in Tecplot 360. Similar sonography map can establish. We select the $60 \%$ stenosis ICA as an example. Fig. (4) is the blue or red contour of horizon velocity component. When the velocity is toward the right it is in red and else is in blue. The black streamlines are drawn to depict the flow tendency more accurate.

Fig. (5) illustrates the distribution of turbulence kinetic energy. The more turbulent of flow the hue of green more dark or deep. It is a simply way to illustrate the change of flow.

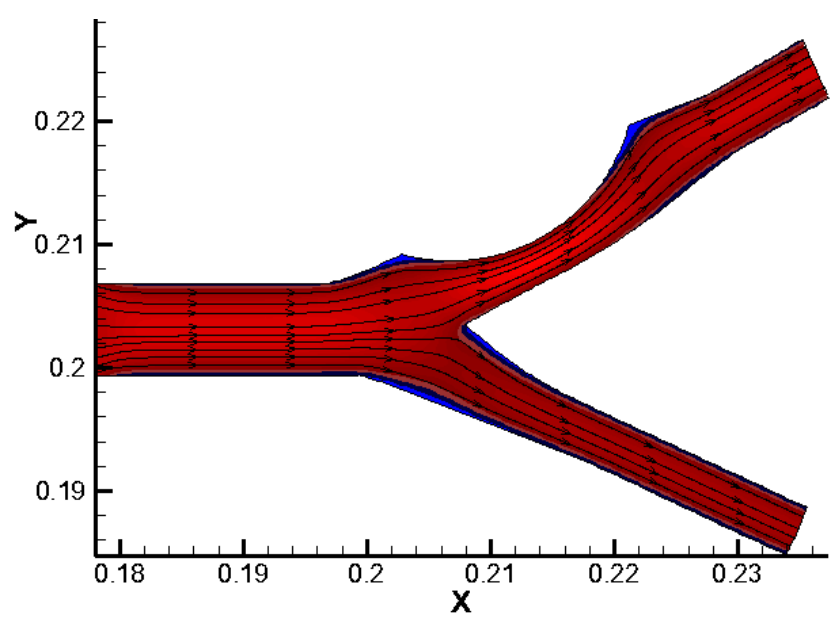

Fig. (4). Filled contour of velocity considering direction and velocity streamline.
Now we get two color flow map, use imaging processing technology, one for velocity direction and magnitude. We mixed the two pictures and color can be joining. The procedure also can be called images fuse. In Fig. (6) the turbulence of flow are consider and turbulence coefficient are indicate as green color to mixed with the velocity. This display method is mocking the ordinary ultrasound instrument display principle.

We can see some place the color mixed greener component or exist the changing of green to cyan, that is the blood stagnant place or highly disturb zone (we can call it dead zone). In Figs. (4 and $\mathbf{6}$ ), we can see that kind zones are always close to the bifurcation or plaques. They can proliferation and deteriorate condition of blood flow. If the dead zone is very big that means very dangerous of the patient.

\section{CONCLUSIONS}

A detailed comparison of effect of different stenosed of ICA on blood flow was done. With the same circumstances, the causing of stagnate zone, blood distribution and the direction of blood field is significant different. Through the simulation we can get more information of the prolife of the stenosis, prediction of vascular complications. Through the

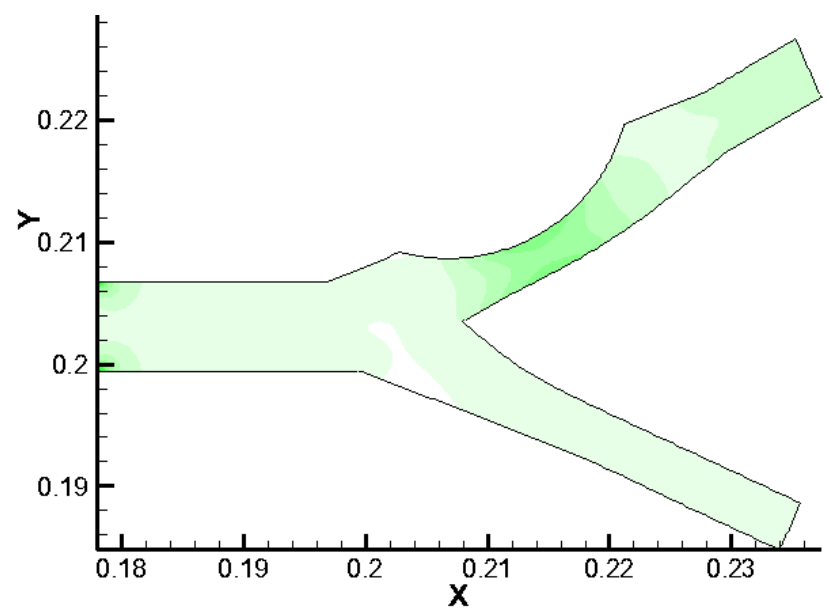

Fig. (5). Filled contour of turbulence kinetic energy.

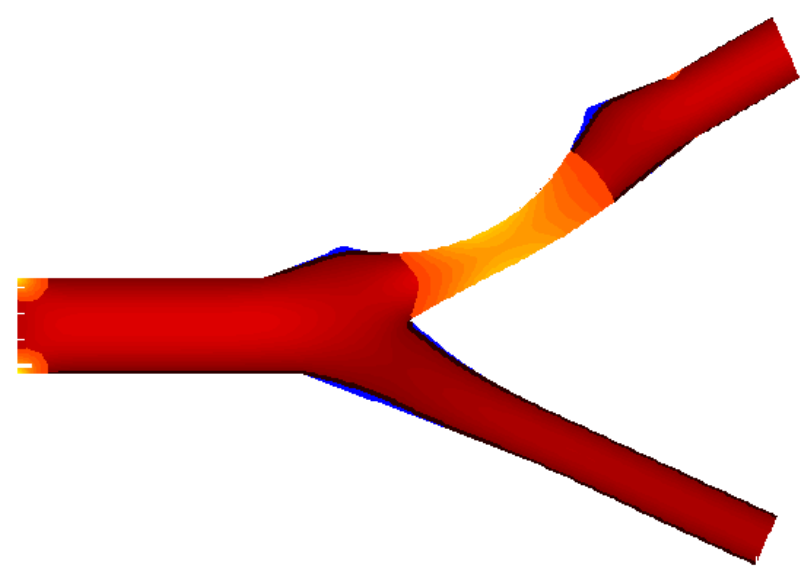

Fig. (6). Simulation of CDFI combines with turbulence information. 
post-process, we mocked the display method of ultrasound and get a color Doppler flow field imaging simulated. The picture may more detailed and familiar of clinics or physicians. That can say, traditional blood measure imaging technology can only get overall flow distribution, but they can use the simulation to aid the diagnosis of vascular stenosis.

Quantification of turbulence near carotid stenoses may provide additional diagnostic information, which could enhance the performance of DUS for diagnosis. These blood properties can be managed by lifestyle changes and drug administration, thus guiding the clinician to better manage high risk patients [22].

\section{CURRENT \& FUTURE DEVELOPMENTS}

The Doppler ultrasound system and associated methods are widely used for monitoring blood flow and detecting emboli and stenosis of vascular. Such ultrasound systems are advantageously used both for diagnostic exams (to determine the presence and significance of vascular disease or dysfunction) and during surgical interventions (to indicate surgical manipulations that produce emboli or alter/interrupt blood flow).

The integration of ultrasound technology and CFD can provide explicit hemodynamic information. The intricate imaging emerge in future may including: 1) 4-D, real-time hemodynamic, 2) patient-specific vascular and blood flow imaging and modeling, 3) Pre-surgery evaluating of vascular deforming and flow change.

\section{ACKNOWLEDGMENTS}

This work was supported by Sichuan Province (P.R.C.) Education Department General Project: (No. 13ZB0012), 2013 teaching reform project of China West Normal University and sponsored research projects of China West Normal University (No.13C002).

\section{CONFLICT OF INTEREST}

The authors declare no conflict of interest.

\section{REFERENCE}

[1] Thrush A, Hartshorne T. Peripheral Vascular Ultrasound: How, Why and When. London: Elsevier 2005.

[2] Fan Y, Jiang W, Deng X, et al. Numerical simulation of pulsatile non-newtonian flow in the carotid artery bifurcation. Acta Mech Sin 2009; 25: 249-55,
[3] Taylor CA, Fonte TA, Zarins CK. Method and system for patientspecific modeling of blood flow. U.S. Patent 8,315,814, Jan 27 , 2011.

[4] Bharadvaj BK, Mabon RF, Giddens DP. Steady Flow in a Model of the Human Carotid Bifurcation. Part I-Flow Visualization. J Biomech 1982; 15: 349-62.

[5] Bharadvaj BK, Mabon RF, Giddens DP. Steady Flow in a Model of the Human Carotid Bifurcation. Part II-Laser Doppler Anemometer Measurements. J Biomech 1982; 15: 363-78.

[6] Swillens A, De Schryver T, Løvstakken L, et al. Two-dimensional flow imaging in the carotid bifurcation using a combined speckle tracking and phase-shift estimator: A Study Based on Ultrasound simulations and in vivo analysis. Ultrasound Med Biol 2010; 36(10): 1722-35

[7] R.D. Henderson, D.A. Steinman, M. Eliasziw, "Effect of contralateral carotid artery stenosis on carotid ultrasound velocity measurements", stroke, vol. 31, pp. 2636-2640, Nov.2000.

[8] Swillens A, Lovstakken A, Kips L, et al. Ultrasound simulation of complex flow velocity fields based on computational fluid dynamics. IEEE Trans Ultrason, Ferroelectr Freq Control 2009; 56: 546-556.

[9] Njemanze PC. Method and system for evaluation of the hemodynamic model in depression for diagnosis and treatment. U.S. Patent 7,942,820, May 17, 2011

[10] Funamoto K, Hayase T, Saijo Y, et al. Numerical analysis of effects of measurement errors on ultrasonic-measurementintegrated simulation. IEEE Trans Biomed Eng 2011; 58: 653-663.

[11] Redano RT. Apparatus for measuring hemodynamic parameters. U.S. Patent 6,814,702, April 27, 2002.

[12] Sharma P, Georgescu B, Ionasec RI, Comaniciu D. Method and system for computational modeling of the aorta and heart. U.S. Patent 8, 224, 640, July 17, 2012.

[13] Thorne ML, Poepping TL. In vitro Doppler ultrasound investigation of turbulence intensity in pulsatile flow with simulated cardiac variability. Ultrasound Med Biol 2009; 35: 1208.

[14] Ford MD, Alperin N, Lee SH, et al. "Characterization of volumetric flow rate waveforms in the normal internal carotid and vertebral arteries. Physiol Meas 2005; 26: 477-88.

[15] Szabo TL. Diagnostic ultrasound imaging: inside out. London: Elsevier 2004.

[16] Khoshniat M, Thorne ML, Poepping TL. Real-time numerical simulation of doppler ultrasound in the presence of nonaxial flow. Ultrasound Med Biol 2005; 31: 519-528.

[17] Haider BH. Method and apparatus for flow parameter imaging. U.S. Patent 8,469,887, June 25, 2013.

[18] Moehring MA, Farnsworth JH, Zachariah AP, Baron, Jr. HJ. Doppler ultrasound method and apparatus for monitoring blood flow and hemodynamics. U.S. Patent 7, 128, 713, 2006.

[19] Mo LYL, Cobbold RSC. "Speckle" in continuous wave doppler ultrasound spectra: a simulation study. IEEE Transact Ultrasonics, Ferroelectr Freq Control 1986; 33; 747-53.

[20] Hoskins P, Martin K, Thrush A. Diagnostic ultrasound physics and equipment. Cambridge: Cambridge University Press 2010.

[21] Younis HF, Kaazempur-Mofrad MR, Chan RC. Hemodynamics and wall mechanics in human carotid bifurcation and its consequences for atherogenesis: investigation of inter-individual variation. Biomech Model Mechanbiol 2004; 3(1): 17-32.

[22] Jabbar AU, Ali RU, Parvez K, et al. Three-Dimensional numerical analysis of pulsatile blood flow around different plaque shapes in human carotid artery. Int J Biosci Biochem Bioinforma 2012; 2(5): 305-08. 